\title{
ANÁLISIS DEL EFECTO DEL HUMEDECIMIENTO EN LA ESTABILIDAD DE UN BORDO DEL RÍO GRIJALVA, EN EL ESTADO DE TABASCO
}

\section{ANALYSIS OF THE EFFECT OF WETTING ON THE STABILITY OF A SIDE SLOPE OF THE GRIJALVA RIVER, IN THE \\ STATE OF TABASCO}

\author{
Romelia Ávila Mondragón \\ ingrome_avila@hotmail.com \\ René Sebastián Mora Ortiz \\ rene.mora@ujat.mx \\ Francisco Magaña Hernández \\ francisco.magana@ujat.mx \\ Sergio Alberto Díaz Alvarado \\ alberto.diaz@ujat.mx
}


Para citar este artículo:

Ávila-Mondragón, R., Mora-Ortiz, R, Díaz-Alvarado, Sergio. (2019). Análisis del efecto del humedecimiento en la estabilidad de un bordo del Río Grijalva, en el estado de Tabasco. Espacio I+D, Innovación más Desarrollo. VIII(21), 63-75. doi: http://dx.doi.org/10.31644/ IMASD.21.2019.a04

\section{RESUMEN}

Durante la época de lluvias se presentan en el estado de Tabasco (México) deslizamientos en los bordos de los ríos que atraviesan la entidad, siendo los de la zona conocida como La Manga de los más afectados. Este artículo presenta el análisis de la evolución de la estabilidad de uno de estos bordos debido a los cambios en el grado de saturación del suelo ( $\mathrm{Sr}$ ) que lo conforma. El objetivo fue determinar el Sr del suelo para el cual el bordo pasa de una condición de seguridad a una de falla. Para lograrlo, primero se realizó una campaña de exploración de campo y ensayos de laboratorio, lo que permitió obtener las características geométricas del bordo, así como las propiedades del suelo que lo constituye. De las muestras inalteradas obtenidas en campo se realizaron ensayos de compresión triaxial variando solo el grado de saturación del suelo. Con los datos obtenidos y mediante el software GeoSlope 2016 se realizaron diversos análisis de la estabilidad. Los resultados muestran que conforme el grado de saturación crece la estabilidad del bordo disminuye. Se comprobó que para el bordo en estudio sobrepasar un grado de saturación de 70 \% implica una condición de peligro de deslizamiento. Por lo tanto, el monitoreo del grado de saturación del suelo que constituye a los bordos permite anticipar su falla y, en consecuencia, hace posible establecer acciones de prevención.

\section{Palabras clave}

Bordo; cohesión; estabilidad; factor de seguridad. 
During the rainy season in the Mexican state of Tabasco there are landslides over the rivers that flow through the state, being some of the most affected area the one called "La Manga". This paper presents the stability analysis evolution of the side slope due to changes over the soils saturation that forms it, going from safety condition to failure condition. In order to achieve this goal, a campaign of field exploration and laboratory tests was first carried out, which allowed obtaining the geometric characteristics of the side slope, as well as the soil properties that constitutes it. From the undisturbed samples obtained in the exploration field, triaxial compression tests were carried out, varying only the degree of saturation of the soil. With the information obtained and through the GeoSlope 2012 software, stability analyses were performed varying only the degree of saturation. The results show that as the degree of saturation increases, the stability of the board decrease. It was found that exceeding $70 \%$ degree of saturation implies a slip hazard condition. Therefore, the monitoring of the soil degree of saturation that constitutes side slopes allows to anticipate its failure and, consequently, makes it possible to establish prevention actions.

\section{Keywords}

Side slope; cohesion; stability; safety factor. 
L

os taludes o laderas, son las superficies inclinadas con respecto a la horizontal que han de adoptar las estructuras de suelo, ya sea de origen natural o producto de la actividad del hombre durante la ejecución de obras civiles (Juárez y Rico, 2005). Los taludes están presentes en obras civiles como presas, excavaciones, túneles, bordos de los ríos, rellenos sanitarios, etc. Históricamente, en México han ocurrido deslizamientos de laderas naturales y artificiales con más frecuencia durante las épocas de lluvia y durante eventos sísmicos. La geología, el relieve, el intemperismo, la erosión y la historia tectónica son factores que condicionan al deslizamiento de los taludes en todo el mundo. Sin embargo, el humedecimiento del material constituyente del talud es quizá uno de los más importantes catalizadores de las fallas estructurales de las laderas. Durante los eventos de precipitación pluvial los taludes están expuestos al avance del agua dentro de su cuerpo por el exceso de infiltraciones prolongadas. Como consecuencia de esto, la resistencia al esfuerzo cortante se reduce rápidamente y aumenta el peso volumétrico del suelo (Cho y Lee, 2001; 2002; Ching-Chuan et al., 2008; Akay, 2016; Sun et al., 2016). Las fallas estructurales de taludes que se presentan durante la época de lluvias lo hacen independientemente del tipo de suelo que los compone y de su geometría. Esto es evidencia de que el humedecimiento del material constitutivo del talud es uno de los principales factores que generan la inestabilidad. Normalmente los deslizamientos de laderas son poco profundos (Xie et al., 2004). Este tipo de fallas están vinculadas con lluvias de regular intensidad pero de periodos prolongados y pueden producir presiones de poro positivas en el cuerpo del talud (FloresBerrones et al., 2003). Las fallas más profundas están vinculadas con la disminución de la succión (Collins y Znidarcic, 2004) y se deben a lluvias muy fuertes y prolongadas (Casagli et al., 2005). Gran parte de los deslizamientos de taludes ocurren en zonas cuyo clima implica lluvias frecuentes durante el año (Tohari et al., 2007; Mora-Ortiz y Rojas-González, 2012; Sun et al., 2017). Por lo anterior, diversos investigadores (Conte y Troncone, 2017; Wang et al., 2018) han trabajado en métodos que intentan predecir la fallas en los taludes.

El estado de Tabasco se encuentra dentro de la zona tropical lluviosa del país, con influencia de ciclones tropicales y nortes. Posee un clima cálido húmedo con lluvias todo el año siendo más abundantes en los meses de junio a octubre (Inegi, s.f.; García Payró, 2015). Tabasco es una llanura de inundación donde convergen diferentes ríos, entre los que destacan el Tonalá, Grijalva y Usumacinta. La entidad se encuentra en la parte baja de la cuenca Grijalva-Usumacinta, cuenca que concentra casi la tercera parte del agua superficial del país. En la capital tabasqueña, Villahermosa, existen diferentes zonas que tradicionalmente presentan inestabilidad de los bordos de los ríos durante las épocas de lluvia. Una de estas zonas es la conocida 
como Acachapan y Colmena. Esta investigación pretende determinar el efecto del humedecimiento del suelo en la inestabilidad de uno de los bordos del río que pasa en dicha zona, específicamente un bordo localizado en la colonia La Manga 2da sección. Se desea identificar el grado de saturación para el cual la estabilidad de este bordo se encuentra en riesgo. El objetivo de este artículo es establecer el primer paso para la creación de un sistema de alerta de deslizamiento de bordos en los ríos de Tabasco.

\section{METODOLOGÍA}

El bordo en estudio se localiza en la colonia la Manga 2da sección, municipio de Centro, Tabasco (Figura1). Se eligió esta zona debido a que se encuentra en uno de los sectores más afectados por la inestabilidad de los bordos, el llamado sector La Manga. Una vez elegida la zona de estudio fue necesario contar con las características geométricas del bordo y con las propiedades del suelo que lo compone. Para lograr lo anterior, se realizó una campaña de reconocimiento de campo donde, mediante equipo topográfico, se determinó el perfil geométrico del bordo. Para obtener las características del suelo constituyente del bordo se extrajeron tres muestras inalteradas (cubos de $20 \mathrm{~cm}$ por lado) mediante el método de pozo a cielo abierto (PCA) a una profundidad de $1.5 \mathrm{~m}$ siguiendo el procedimiento marcado por la norma NMX-C-416-ONNCCE-2003.

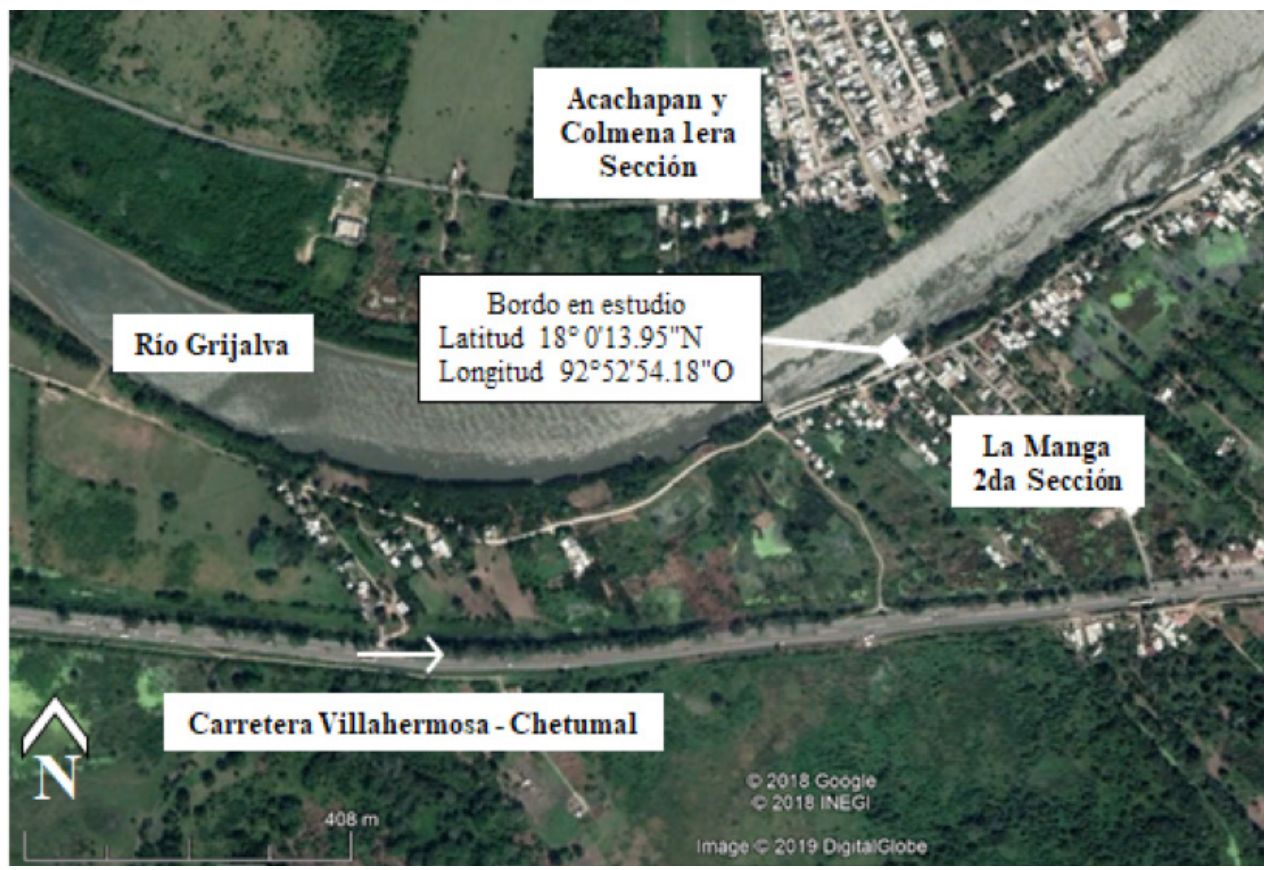

Figura 1. Ubicación del bordo en estudio 
La Tabla 1 muestra las propiedades básicas del suelo determinadas en el laboratorio de mecánica de suelos de la Universidad Juárez Autónoma de Tabasco (UJAT). El límite líquido (LL) se determinó con el procedimiento de la copa de Casagrande; el límite plástico (LP) con el método de Atterberg, el índice de plasticidad (IP) con la diferencia aritmética entre el LL y el LP. Los pesos específicos de la masa del suelo y relativo de sólidos se terminaron con los ensayos de laboratorio que llevan su nombre. Todos los ensayos se realizaron siguiendo el procedimiento descrito en la norma NMX-C-416-ONNCCE-2003.

\section{Tabla 1}

Características básicas y clasificación del material

\begin{tabular}{cccc}
\hline Límite líquido (LL) & $76 \%$ & $\begin{array}{c}\text { Peso específico relativo de } \\
\text { sólidos }\end{array}$ & 2.64 \\
Límite Plástico (LP) & $41 \%$ & Clasificación S.U.C.S* & $\begin{array}{c}\text { MH (Limo de alta } \\
\text { plasticidad con arena) }\end{array}$ \\
$\begin{array}{c}\text { Índice de Plasticidad } \\
(\mathrm{IP})=\mathrm{LL}-\mathrm{LP}\end{array}$ & $35 \%$ & Grado de saturación (Sr) & $42.20 \%$ \\
$\begin{array}{c}\text { Peso específico de la } \\
\text { masa de suelo }(\gamma \mathrm{m})\end{array}$ & $16.83 \mathrm{kN} / \mathrm{m}^{3}$ & & 0.77 \\
\hline
\end{tabular}

* Sistema Unificado de Clasificación de Suelos

Como se observa en la Tabla 1, el suelo posee un alto valor del índice de plasticidad ( IP $\geq 18$ ), lo que significa en términos ingenieriles que es un material susceptible a disminuciones de resistencia cuando se humedece.

Los principales parámetros del suelo que rigen la estabilidad de un talud o bordo son dos: el ángulo de fricción $(\varphi)$ y la cohesión (c). La magnitud de esta última está estrechamente ligada al contenido de agua del suelo, de manera que a grandes contenidos de agua los valores de cohesión son mínimos. Es decir, entre más agua tenga el suelo, menor será el valor de la cohesión y, por consiguiente, menor será la estabilidad del bordo. Por todo lo anterior, monitorizar los valores de cohesión y su respectivo impacto en la estabilidad del talud permite identificar el valor crítico de este parámetro para el cual el bordo pasa de un estado de seguridad estructural a la condición de falla. La mejor forma de determinar los valores de la cohesión (c) y del ángulo de fricción ( $\varphi$ ) es mediante el ensayo de laboratorio conocido como Triaxial consolidado-drenado siguiendo el procedimiento clásico descrito por Juárez y Rico (2005).

Dado que se desea conocer la evolución de la estabilidad del bordo del río según se va humedeciendo el suelo que lo constituye, es necesario conocer los cambios en la magnitud de la cohesión a medida que la cantidad de agua aumenta en el suelo. Para ello, se realizaron ensayos de com- 
presión triaxial en distintas muestras de este suelo, variando únicamente su contenido de agua. De las muestras inalteradas que se extrajeron durante la campaña de reconocimiento se labraron especímenes cilíndricos de $38 \mathrm{~mm}$ de diámetro y $76 \mathrm{~mm}$ de alto (Figura 2). El labrado de muestras es el proceso a través del cual mediante herramienta menor como navajas y cúters, se extraen de muestras de suelo inalteradas (cubos de $20 \mathrm{~cm}$ por lado) especímenes cilindros, con la finalidad de realizar algún ensayo de laboratorio.

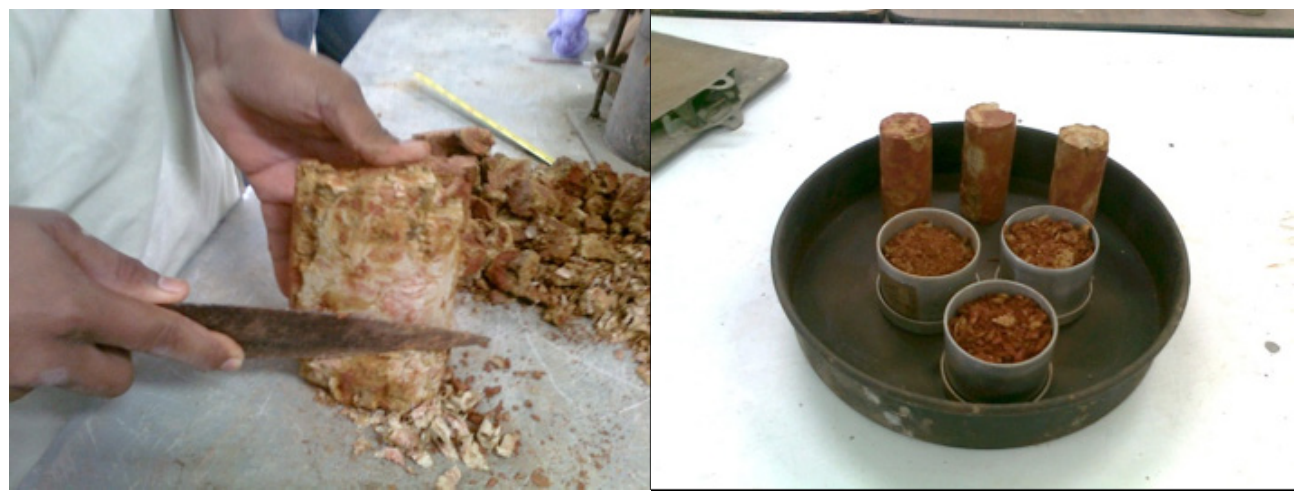

Figura 2. Labrado de especímenes para el ensayo triaxial

Para obtener el valor de la cohesión correspondiente al estado más seco del suelo, se sometió a tres especímenes a un secado progresivo al aire libre evitando el contacto directo de los rayos del sol para prevenir agrietamientos. Posteriormente, se realizó un ensayo triaxial a los especímenes. De esta manera se obtuvo el valor de la cohesión para el estado seco, mismo que se alcanza durante la época de estiaje.

Para conocer la variación de la cohesión del suelo debido a diferentes contenidos de agua, se labraron especímenes y partiendo de la condición seca (descrita anteriormente) se humedecieron de tres en tres mediante un leve rocío de agua con la ayuda de un atomizador. Para garantizar la homogeneidad de la humedad en las muestras después de humedecerlas, se colocaron en recipientes cerrados herméticamente durante 24 horas. Al final de este procedimiento se obtuvieron siete grupos de tres especímenes, cada grupo con un grado de saturación distinto (Tabla 2). Desde el estado más seco hasta el estado más húmedo, se realizaron diversos ensayos triaxiales, lo que permitió conocer la evolución de la cohesión en el suelo en estudio.

\section{Tabla 2}

Cohesión para diferentes grados de saturación

$\begin{array}{ccc}\text { Grupos de } 3 \text { Especímenes } & \text { Grado de saturación }(\mathrm{Sr}) & \text { Cohesión }\left(\mathrm{kN} / \mathrm{m}^{2}\right) \\ 1 & 8 \% & 57.45\end{array}$




\begin{tabular}{llc}
2 & $14 \%$ & 48.77 \\
3 & $22 \%$ & 33.41 \\
4 & $41 \%$ & 25.68 \\
5 & $67 \%$ & 9.27 \\
6 & $83 \%$ & 3.98 \\
7 & $95 \%$ & 0.48 \\
\hline
\end{tabular}

Para evaluar la estabilidad del bordo se utilizó el software Geo-Slope (2016). Éste es un programa de cómputo internacional utilizado para evaluar la estabilidad de taludes de roca y suelo en diferentes condiciones de carga y nivel freático (Gofar et al., 2009; Leung et al., 2017; Munro y Mohajerani, 2018). El programa requiere como datos de entrada la geometría del talud, condiciones del nivel freático en la zona, la cohesión, el peso específico del material constituyente del talud $\left(16.83 \mathrm{kN} / \mathrm{m}^{3}\right)$ y el ángulo de fricción del suelo $\left(24^{\circ}\right)$. Toda esta información fue recopilada durante la exploración de campo y mediante ensayos de laboratorio.

\section{RESULTADOS}

Una vez recopilada la información geométrica del bordo y las características del suelo, se analizó su estabilidad. Este análisis consiste en cuantificar las fuerzas que producen la inestabilidad, así como aquellas que se oponen a la falla. Al cociente entre las fuerzas que se oponen a la falla y aquellas que la provocan, se le conoce como factor de seguridad (Fs) (Juárez y Rico, 2005; Xiao, 2018). Si el valor numérico de este factor es menor a uno (FS $\leq$ 1), se considera que el bordo está en condición de falla. Por otro lado, si se cumple que Fs $\geq 1.5$, se estima que el bordo es seguro. Si el Fs se encuentra entre los dos límites antes mencionados $(1<$ FS $<1.5)$ se considera que el bordo está en riesgo. El método utilizado en esta investigación para calcular el fs fue el método de las dovelas de Fellenius (Juárez y Rico, 2005). Este método ha sido ampliamente estudiado he implementado en el análisis de taludes de suelo y ha mostrado un excelente desempeño. La Figura No 3 muestra el análisis de estabilidad del bordo para los distintos valores de cohesión mostrados en la Tabla 2. En cada análisis se observa una malla en la cual se muestra el valor mínimo del Fs. Como se muestra en la Figura 3, el factor de seguridad más elevado corresponde a la condición de cohesión más alta, es decir, al bordo más seco. Puede verse que conforme el grado de saturación aumenta en el suelo constituyente del bordo, el factor de seguridad disminuye (Figura 4). Según los resultados obtenidos puede verse que la estabilidad del bordo está garantizada ( FS $\geq 1.5$ ) siempre y cuando el grado de saturación del suelo que lo compone sea menor a 70 \% (Figura 4). 


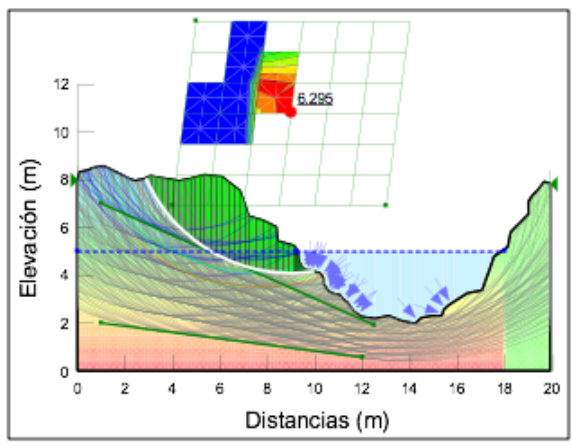

Grado de saturación $=8 \%$

Cohesión $=57.45 \mathrm{kN} / \mathrm{m}^{2}$

$\mathrm{FS}=6.295$

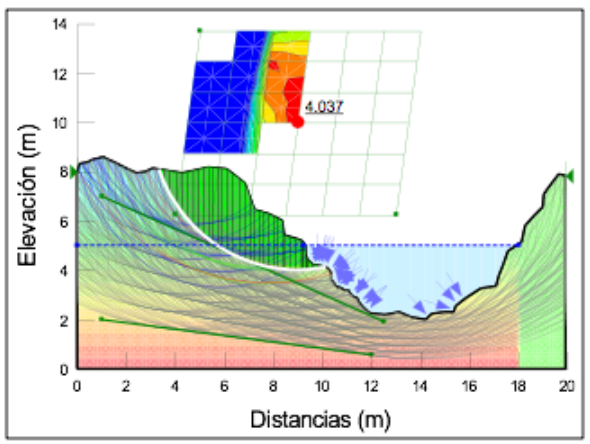

Grado de saturación $=22 \%$

Cohesión $=33.42 \mathrm{kN} / \mathrm{m}^{2}$

$\mathrm{FS}=4.037$

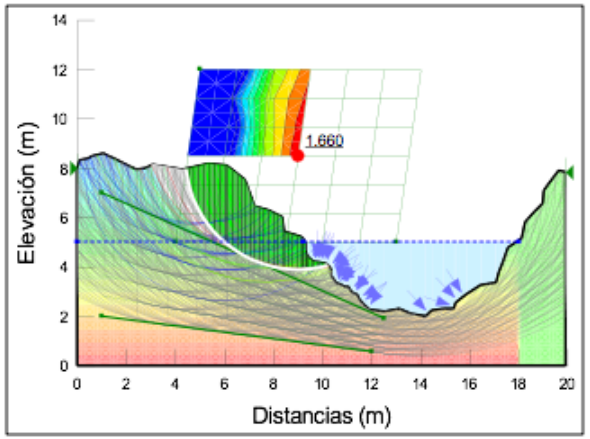

Grado de saturación $=67 \%$

Cohesión $=9.27 \mathrm{kN} / \mathrm{m}^{2}$

$\mathrm{FS}=1.660$

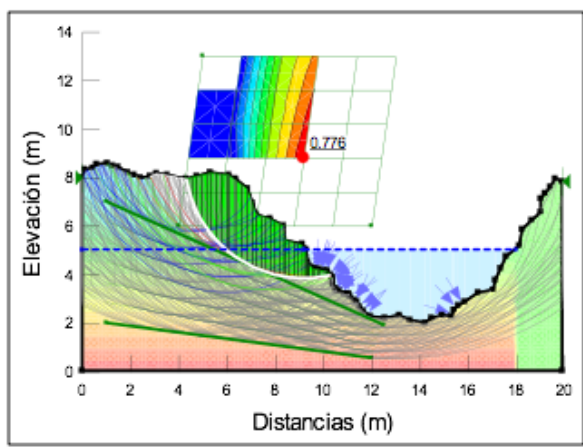

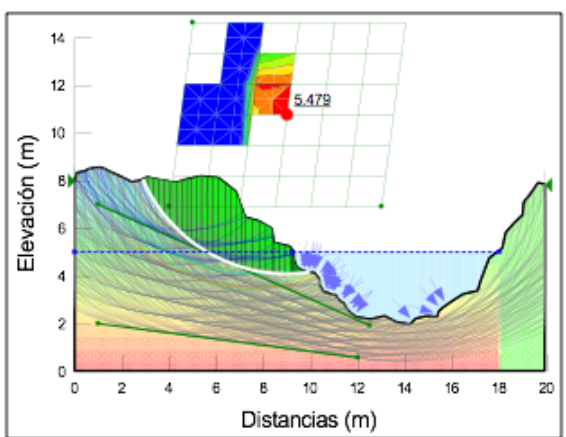

Grado de saturación $=14 \%$

Cohesión $=48.77 \mathrm{kN} / \mathrm{m}^{2}$

$\mathrm{FS}=5.479$

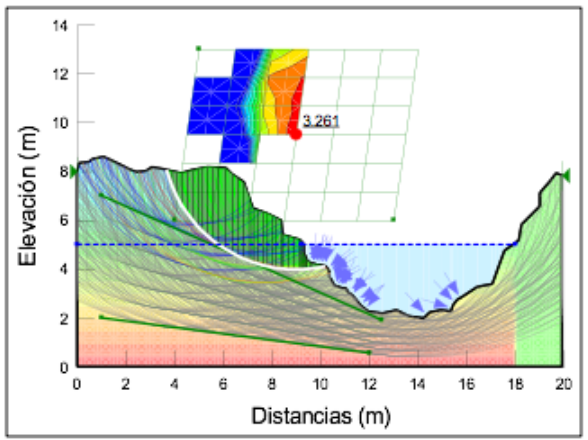

Grado de saturación $=41 \%$

Cohesión $=25.68 \mathrm{kN} / \mathrm{m}^{2}$

$\mathrm{FS}=3.261$

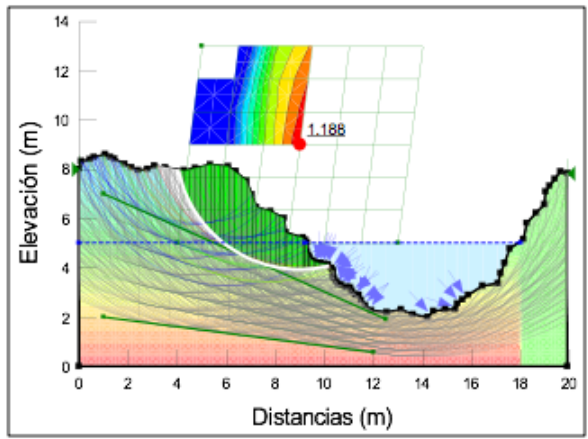

Grado de saturación $=83 \%$

Cohesión $=3.98 \mathrm{kN} / \mathrm{m}^{2}$

$\mathrm{FS}=1.188$

Grado de saturación $=95 \%$

Cohesión $=0.48 \mathrm{kN} / \mathrm{m}^{2}$

$\mathrm{FS}=0.776$

Figura 3. Labrado de especímenes para el ensayo triaxial 


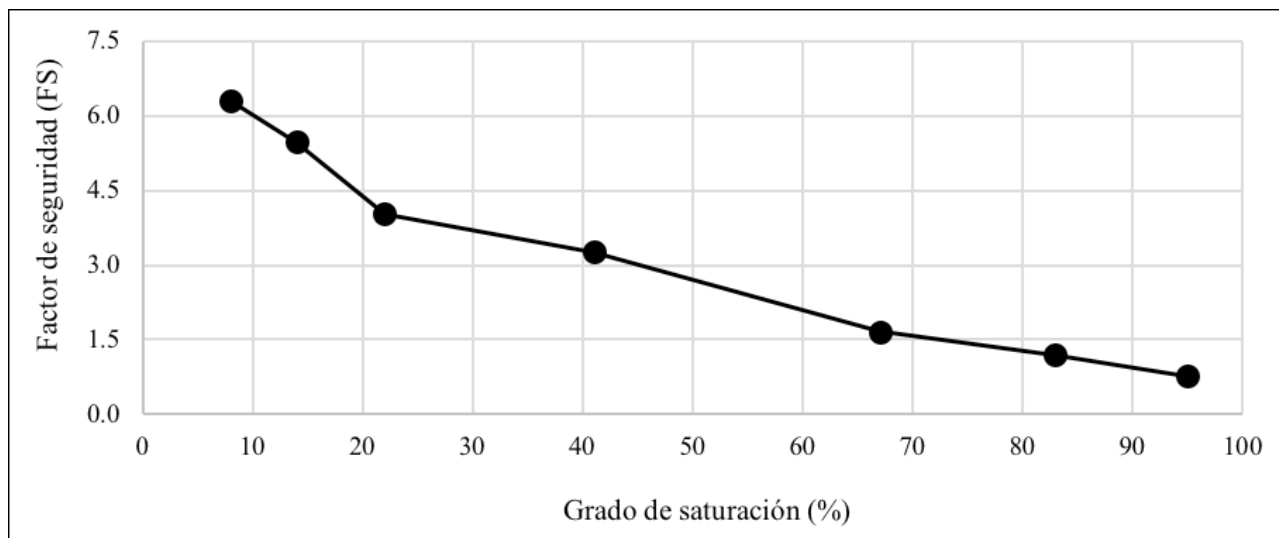

Figura 4. Evolución del factor de seguridad con los cambios en el grado de saturación del suelo

De todo lo anterior se desprenden dos conclusiones:

- Si la precipitación pluvial es suficiente como para situar al grado de saturación del suelo entre un $70 \%$ y $85 \%$, el bordo se encuentra en una situación de riesgo de falla $(1<\mathrm{FS}<1.5)$;

- $\quad$ Si el humedecimiento del bordo sitúa al grado de saturación por encima de los $85 \%$, se presentará la falla del bordo (FS $\leq 1)$.

\section{DISCUSIÓN}

En la estabilidad de los bordos influyen diferentes factores como el tipo de material constituyente, la geología, el intemperismo, la erosión (por agua o aire), etc. Sin embargo, en este estudio nos hemos enfocado solo en el humedecimiento del material que compone al bordo. Lo anterior debido a que la evidencia experimental publicada por investigadores como Mora-Ortiz y Rojas-Gonzalez, 2012; Conte et al., 2017; Sun et al., 2017, por mencionar algunos, señala al humedecimiento del suelo como uno de los principales factores que provocan la inestabilidad en taludes localizados cerca o lejos de los ríos. Este trabajo no descarta el efecto desestabilizador de otros agentes, simplemente pretende poner en evidencia que la saturación del suelo que compone al bordo en estudio por sí sola es capaz de provocar la falla. Una línea de investigación futura consiste en incorporar a este análisis todos los posibles agentes que contribuyen a la inestabilidad de los bordos.

Los resultados presentados en esta investigación han demostrado que el humedecimiento progresivo del suelo constituyente del bordo estudiado ocasiona la disminución del factor de seguridad. Después de la inundación en el estado de Tabasco que se presentó en el año 2007 fue necesario reparar 43 bordos localizados solo en la capital tabasqueña. Lo anterior representó 
una inversión de 152.4 millones de pesos. Al año siguiente, después de un periodo intenso de lluvias (octubre-noviembre), se repararon 53 bordos, esta vez con un costo de 200 millones de pesos (Marí, 2009). Los resultados obtenidos en esta investigación muestran (al menos para el bordo en estudio) que cuando el suelo alcanza un grado de saturación superior a 85 $\%$ la condición de estabilidad del bordo es de riesgo. Alcanzar este grado de saturación en el estado de Tabasco no es difícil, ya que la entidad se encuentra en la parte baja de la cuenca Grijalva-Usumacinta (la cual, como ya se dijo, concentra casi la tercera parte del agua superficial de México), además se localiza en la zona tropical lluviosa del país, con influencia de ciclones tropicales y nortes. En la entidad se presentan lluvias todo el año, siendo la precipitación media anual de $2250 \mathrm{~mm}$, aunque las lluvias más abundantes se presentan durante los meses de junio a octubre (INEGI, s.f.).

Llama la atención que a pesar de los datos sobre las fallas de los bordos en el estado de Tabasco no exista hoy en día un estudio que permita definir las zonas más propensas a las fallas durante la época de lluvias. Es decir, no existe un mapa de peligrosidad de deslizamiento de los bordos. La ciudad de México cuenta con mapas de peligrosidad geológica y de inestabilidad de laderas, además existen mapas de zonificación geotécnica y zonificación sísmica (Protección Civil, s.f.)

\section{CONCLUSIONES}

Debido a su situación climatológica en el estado de Tabasco se presentan lluvias durante todo el año siendo más abundantes durante los meses de junio a noviembre. Durante estos periodos de lluvia consistentemente se han registrado fallas en los bordos de los ríos que llegan a la capital del estado de Tabasco. La presente investigación se centró en analizar el efecto solo del humedecimiento del suelo en la estabilidad del bordo. Se comprobó que para un bordo localizado en la zona conocida como La Manga, el humedecimiento del suelo que compone al bordo es uno de los principales factores que provocan la inestabilidad. Se constató que a medida que el grado de saturación aumenta el factor de seguridad (FS) disminuye desde un estado seguro (FS $\geq$ 1.5) hasta un estado de falla ( FS $\leq 1)$. Se pudo comprobar que para el bordo en estudio sobrepasar un grado de saturación (Sr) de 70 \% representa una condición de peligro de falla. Es decir, mediante el monitoreo de las lluvias y del grado de saturación del suelo que forma a los taludes es posible anticipar la falla de los mismo. El presente estudio muestra una metodología simple que puede ser la base para elaborar un mapa de peligrosidad de falla de bordos. Debido a que todos los bordos cambian en sus características geométricas y propiedades de los suelos que los componen, es necesario repetir el proceso 
aquí señalado para todos los bordos que se deseen monitorear. Lo anterior permitirá a las autoridades pertinentes tomar acciones para garantizar la integridad de personas y de las estructuras cercanas a los bordos. 


\section{REFERENCIAS}

Akay, O. (2016). Slope stabilisation using EPS block geofoam with internal drainage system. Geosynthetics International, 23 (1), 9-22.

Casagli N., Dapporto S., Ibsen M.L., Tofani V. y Vannocci P. (2005). Analysis of the Landslides Triggering Mechanism During the Storm of 2oth-21st November 2000 in Northern Tuscany. Landslides, 3, 13-21.

Ching-Chuan H., Chien-Li L., Jia-Shiun J. y Lih-Kang H. (2008). Internal Soil Moisture Response to Rainfall-Induced Slope Failures and Debris Discharge. Engineering Geology, 101, 134-145.

Cho S.E. y Lee S.R. (2001). Instability of Unsaturated Soil Slopes Due to Infiltration. Computers and Geotechnics, 28, 185-208.

Cho S.E. y Lee S.R. (2002). Evaluation of Surficial Stability for Homogeneous Slopes Considering Rainfall Characteristics. Journal of Geotechnical and Geoenvironmental Engineering, 128, 756-763.

Collins B.D. y Znidarcic D. (2004). Stability Analyses of Rainfall Induced Landslides. Journal of Geotechnical and Geoenvironmental Engineering, 130, 362-372.

Conte, E. and Troncone, A. (2017). A simplified method for predicting rainfallinduced mobility of active landslides. Landslides, 14(1), 35-45.

Flores- Berrones R., Alva-García F. y Li-Liu X. (2003). Efecto del flujo de agua en la estabilidad de taludes. Ingeniería hidráulica en México, 18, 35-52.

García Payró, O. (2015). Historia y Geografía de Tabasco. Editorial Santillana.

Geo-Slope International. (2016). GeoStudio SLOPE/W (2012 edition). Recuperado de: http://www.geo-slope.com/products/slopew.aspx.

Gofar N., Lee M.L. y Kasim A. (2009). Extreme rainfall for slope stability evaluation. Prediction and Simulation Methods for Geohazard Mitigation. Oka, Murakami \& Kimoto (eds). Taylor \& Francis Group, London, 499-503.

INEGI (s.f.). Mapa climático de México. Clima de Tabasco. Recuperado de: http://gaia.inegi.org.mx/mdm6/?v=bGFoOjIzLjg4NzQ2LGxvbjotMTA xLjUwMDAwLHo6MSxsOmMoMDF8YzQwNHxjNDA3fGMoMTB8YzQ $\mathrm{xN} 3 x j$ NDE4\&layers $=\mathrm{c} 401, \mathrm{c} 404, \mathrm{c} 407, \mathrm{c} 410, \mathrm{c} 417, \mathrm{c} 418$. Fecha de consulta: 23 de mayo del 2019.

Juárez-Badillo E. y Rico-Rodríguez A. (2005). Mecánica de Suelos. Fundamentos de la Mecánica de Suelos. Tomo 1. Editorial Limusa S.A. de C.V. Grupo Noriega Editores. México.

Leung A.K., Kamchoom V. y Ng C.W.W. (2017). Influences of root-induced soil suction and root geometry on slope stability: a centrifuge study. Canadian Geotechnical Journal, 54, 291-303.

Marí C. (2009). Minimiza CNA fallas en bordos. Villahermosa, Tabasco, México. Fondo para la comunicación y educación ambiental. Recuperado de: 
https://agua.org.mx/minimiza-cna-fallas-en-bordos/. Fecha de consulta: 24 de enero del 2019.

Mora-Ortiz R. y Rojas-González E. (2012). Efecto de la saturación en el deslizamiento de talud en la comunidad San Juan de Grijalva, Chiapas. Ingeniería Investigación y Tecnología, 13, 55-68.

Munro M.C. y Mohajerani A. (2018). Slope stability evaluation of iron ore fines during marine transport in bulk carriers. Canadian Geotechnical Journal, 55, 258-278.

Norma NMX-C-416-ONNCCE-2003. Industria de la Construcción. Muestreo de Estructuras Térreas y Métodos de Prueba. Diario Oficial de la Federación. México, D.F. a 03 de octubre de 2003.

Protección Civil (s.f.) Mapas de Peligros Geológicos. Gobierno de la Ciudad de México. Recuperado de: http://data.proteccioncivil.cdmx.gob.mx/ mapas_sgm/mapas_sgm2.html. Fecha de consulta: 23 de mayo del 2019.

Sun, G., Yang, Y., Cheng, S. and Zheng, H. (2017). Phreatic line calculation and stability analysis of slopes under the combined effect of reservoir water level fluctuations and rainfall. Canadian Geotechnical Journal, 54(5), 631-645.

Sun, G., Zheng, H., Tang, H. and Dai, F. (2016). Huangtupo landslide stability under water level fluctuations of the Three Gorges reservoir. Landslides, 13(5), 1167-1179.

Tohari A., Nishigaki M. y Komatsu M. (2007). Laboratory Rainfall Induced Slope Failure with Moisture Content Measurement. Journal of Geotechnical and Geoenvironmental Engineering, 133, 575 .

Wang, Chien-Chih., Chang, Wen-Jong., Huang, An-Bin., Chou, Shih-Hsun. and Chien, Yu-Chun. (2018). Simplified monitoring and warning system against rainfall-induced shallow slope failures. Canadian Geotechnical Journal, 55, 1421-1432.

Xiao, S., Guo, W.D. and Zenga, J. (2018). Factor of safety of slope stability from deformation energy. Canadian Geotechnical Journal, 55(2), 296-302.

Xie M., Esaki T. y Cai M. (2004). A Time-Space Based Approach for Mapping Rainfall-Induced Shallow Landslide Hazard. Environmental Geology, 46, 840-850. 